\title{
Self-Maintaining Defect/Energetic Droplets from Two Interacting Bose-Einstein Condensates
}

\author{
V. DzhunushalieV* \\ Dept. Theor. and Nucl. Phys., KazNU, Almaty, 010008, Kazakhstan \\ (Received December 27, 2012; in final form June 24, 2013)
}

\begin{abstract}
We consider two interacting Bose-Einstein condensates with different kinds of the potential energy of interaction of the condensates: (a) the standard potential, (b) the potential has a positive three-body and a negative two-body scattering terms, and (c) the potential has a positive four-body and a negative three-body scattering terms for the first Bose-Einstein condensate and a positive three-body and a negative two-body scattering terms for the second Bose-Einstein condensate. It is shown that in these cases there exist stationary regular spherically symmetric solutions. Physically such solution is either a defect or an energetic droplet created by the condensates. The defect is a cavity filled with one Bose-Einstein condensate on the background of another Bose-Einstein condensate. The droplet is an object with zero energy density at the infinity. For (a) and (b) cases the obtained objects are supported by a constant external trapping potential and for (c) case the droplet is a self-maintaining object without any external potential. The possibility of construction of an elementary logic qubit device on the basis of this droplet is discussed.
\end{abstract}

DOI: 10.12693/APhysPolA.124.629

PACS: 03.75.Lm, 03.75.Nt

\section{Introduction}

Usually two interacting Bose-Einstein condensates (BEC's) are described by equations where the potential energy of interaction of the condensates has terms of 2 th and 4th orders [1]. But in Ref. [2] it was shown that the Hamiltonian of two interacting BEC's may have the interactions terms with both 6 th and 4 th orders. There was shown that the term of 6 th order can be positive and the term of 4 th order can be negative. Here we would like to consider three different cases of two interacting BEC's: (a) both BEC's have 2 th and 4th orders scattering terms and interact with a constant external potential; (b) both BEC's have positive three-body and negative two-body scattering terms in the potential and interact with a constant external potential; (c) one BEC is as in the previous item but another BEC has positive hypothesized four-body and negative three-body scattering terms and they do not interact with any external potential.

We will show that in such coupled equations set (describing two interacting BEC's) regular solutions appear. From our point of view such solutions describe either defect or a droplet (with zero energy density at the infinity) created by two interacting BEC's. We will show that: in the case (a) we have a defect supported by a constant external potential; in (b) we have a droplet trapped by a constant external potential and in (c) we have a droplet without any support of an external potential. The defect means that we have a cavity on the background of the space filled with a first kind of BEC. The first kind of BEC has a constant energy density at the infinity. The

*e-mail: vdzhunus@krsu.edu.kg cavity is filled with another BEC whose energy density asymptotically is zero. The droplet means that we have an object filled with two BEC's with both asymptotically zero energy densities.

In Ref. [2] it is shown that if three-body term is repulsive (the interactions terms of 6th order in our language) and two-body term is attractive (the interactions terms of 4th order in our language) then the conclusion is made: "Since the two-body contribution to the ground state energy of a dilute Bose gas is negative, the three-body collisions in the regime where $g_{3}>0$ could lead to the stabilization of the system. What is particularly interesting for such a system is that a boson droplet a boselet could become selfbound and the trapping potential is not required anymore to keep the particles together". Below we will show that such self-maintaining configuration (droplet or boselet) does exist as the solution of Eqs. (34), (35) if one BEC has a positive four-body and negative three-body scattering terms and another BEC has a positive three-body and negative two-body scattering terms.

At the present time the interaction between two interacting BEC's is widely investigated. In Ref. [3] the dynamics of two-component atomic Bose gases initially in a mixture encountering a sudden quench of the inter-species interactions is investigated. The authors of Ref. [4] consider the creation of stable, stationary closed vortex loops in two cold atom BEC's. In Ref. [5] the vortex structures of an elongated two-component BoseEinstein condensate is calculated. In Ref. [6] the coupled Gross-Pitaevskii (GP) equations describing the dynamics of two hyperfine states of the Bose-Einstein condensates are investigated and the integrability condition for the propagation of bright vector solitons is deduced. In Ref. [7] the authors investigate the combined soli- 
ton solutions of two-component Bose-Einstein condensates with external potential. The results show that the intraspecies (interspecies) interaction strengths and the external trapped potential clearly affect the formation of darkdark, brightbright, and darkbright soliton solutions in different regions. In Ref. [8] the localized nonlinear matter waves in the two-component Bose-Einstein condensates with time- and space-modulated nonlinearities analytically and numerically is investigated. The dynamics of these matter waves, including the breathing solitons, quasibreathing solitons, resonant solitons, and moving solitons, is discussed. In Ref. [9] sound waves in two-component Bose-Einstein condensates are investigated and there is proposed a new method of wave generation which is based on a fast change of the inter-species interaction constant. In Ref. [10] stable skyrmions in two component Bose-Einstein condensates are considered. In Ref. [11] the authors report the numerical realization of robust 2 - component structures in $2 \mathrm{D}$ and 3D BEC's with non-trivial topological charge in one component. The vortex - bright solitary waves are found to be very robust in both in the homogeneous medium and in the presence of parabolic and periodic external confinement. In Ref. [12] a family of exact vector-soliton solutions for the coupled nonlinear Schrödinger equations with tunable interactions and harmonic potential is presented.

In this work we investigate the possibility of the existence of stationary solutions for two interacting BEC's with and without any external trapping potential.

Our goal here is to show that equations describing two interacting BEC's have 3-dimensional spherically symmetric stationary solutions. We will: (a) obtain solutions with and without an external trapping potential; (b) study BEC's with two, three and four-body scattering terms; (c) show that droplet solutions exist if corresponding equations have scattering terms with different signs: one term should be attractive and another one repulsive one. Physically it means that desired solutions are an equilibrium between attractive and repulsive interactions. Remarkable that such solutions cannot exist for one BEC following to the Derrick theorem [13]. This theorem states that a regular solution (with finite energy) for a nonlinear scalar field (where the potential energy has only a global minimum) may exist in $1+1$ dimension only (kink). In our case we have two interacting BEC's and the potential energy has two global and two local minima that ensures the existence of the required solutions.

\section{Defect solution with two-body scattering terms and external trapping constant potential}

In this section we consider a defect solution. The solution describes the defect filled with one kind of BEC and placed in the space filled with another kind of BEC.

Considering a two-component BEC, the behavior of the condensates that are prepared in two hyperfine states can be described at sufficiently low temperatures by the two-coupled GP equation of the following form [2]:

$$
\begin{aligned}
& \mathrm{i} \hbar \frac{\partial \tilde{\psi}_{1}}{\partial t} \\
& \quad=\left(-\frac{\hbar^{2}}{2 m_{1}} \nabla^{2}+U_{11}\left|\tilde{\psi}_{1}\right|^{2}+U_{12}\left|\tilde{\psi}_{2}\right|^{2}+V\right) \tilde{\psi}_{1}, \\
& \mathrm{i} \hbar \frac{\partial \tilde{\psi}_{2}}{\partial t} \\
& \quad=\left(-\frac{\hbar^{2}}{2 m_{2}} \nabla^{2}+U_{22}\left|\tilde{\psi}_{2}\right|^{4}+U_{21}\left|\tilde{\psi}_{1}\right|^{2}+V\right) \tilde{\psi}_{2},
\end{aligned}
$$

where the condensate wave functions are normalized by particle numbers $N_{i}=\int\left|\tilde{\psi}_{i}\right|^{2} \mathrm{~d} V ; V$ is an external trapping potential. The resulting equations for the wave functions $\psi_{1,2}(\boldsymbol{r}, t)$ in dimensionless form can be written as

$$
\begin{aligned}
& \mathrm{i} \frac{\partial \psi_{1}}{\partial t}=\left(-\nabla^{2}+u_{11}\left|\psi_{1}\right|^{2}+u_{12}\left|\psi_{2}\right|^{2}+v\right) \psi_{1}, \\
& \mathrm{i} \frac{\partial \psi_{2}}{\partial t}=\left(-k \nabla^{2}+u_{22}\left|\psi_{2}\right|^{2}+u_{21}\left|\psi_{1}\right|^{2}+v\right) \psi_{2} .
\end{aligned}
$$

Here $k=m_{1} / m_{2}$ and we redefined $t / t_{0} \rightarrow t ; \boldsymbol{r} / l_{0} \rightarrow \boldsymbol{r}$; $t_{0}=\frac{2 m_{1}}{\hbar \psi_{1}^{2 / 3}(0)} ; \quad l_{0}=\psi_{1}^{-1 / 3}(0) ; \quad \psi_{1,2}=\frac{\tilde{\psi}_{1,2}}{\psi_{1}(0)} ; u_{i i}=$ $\frac{2 m_{1} U_{i i} \psi_{1}^{4 / 3}(0)}{\hbar^{2}} ; u_{12}=\frac{2 m_{1} U_{12} \psi_{1}^{4 / 3}(0)}{\hbar^{2}}$ and $v=\frac{2 m_{1} V}{\hbar^{2} \psi_{1}^{2 / 3}(0)}$. For the simplicity we will consider the case $k=1$. We are searching for a static spherical symmetric solution: $\psi_{1,2}(\boldsymbol{r}, t)=\mathrm{e}^{-E_{1,2} t} \psi_{1,2}(r)$. In this case Eqs. (3), (4) are

$$
\begin{aligned}
& \psi_{1}^{\prime \prime}+\frac{2}{r} \psi_{1}^{\prime}=\psi_{1}\left[\lambda_{3} \psi_{2}^{2}+\lambda_{1}\left(\psi_{1}^{2}-\mu_{1}^{2}\right)\right], \\
& \psi_{2}^{\prime \prime}+\frac{2}{r} \psi_{2}^{\prime}=\psi_{2}\left[\lambda_{3} \psi_{1}^{2}+\lambda_{2}\left(\psi_{2}^{2}-\mu_{2}^{2}\right)\right],
\end{aligned}
$$

where $\lambda_{i}=u_{i i}, i=1,2 ; \lambda_{i} \mu_{i}^{2}=E_{i}-v ; \lambda_{3}=u_{12}=u_{21}$. Equations $(5),(6)$ are written in the form which is convenient for the numerical calculations. For the numerical calculations we choose the boundary conditions and parameters values as follows:

$$
\begin{aligned}
& \lambda_{1}=0.1 ; \quad \lambda_{2}=1 ; \quad \lambda_{3}=1 ; \\
& \psi_{1}(0)=1 ; \quad \psi_{1}^{\prime}(0)=0 ; \\
& \psi_{2}(0)=\sqrt{0.6} ; \quad \psi_{2}^{\prime}(0)=0 .
\end{aligned}
$$

The solution is searched as the nonlinear eigenvalue problem: $\mu_{1,2}$ are eigenvalues and corresponding functions $\psi_{1,2}$ are eigenfunctions. We solve equations set (5), (6) numerically. The profiles of functions $\psi_{1,2}$ in Fig. 1 are presented.

The asymptotic behavior of the functions $\psi_{1,2}$ is the following:

$$
\begin{gathered}
\psi_{1} \approx \mu_{1}-\psi_{1, \infty} \frac{\mathrm{e}^{-r \sqrt{2 \lambda_{1} \mu_{1}^{2}}}}{r}, \\
\psi_{2} \approx \psi_{2, \infty} \frac{\mathrm{e}^{-r \sqrt{\mu_{1}^{2}-\lambda_{2} \mu_{2}^{2}}}}{r}, \\
\text { where } \psi_{1,2, \infty} \text { are constants. }
\end{gathered}
$$

The dimensionless energy density has the form

$$
\varepsilon\left(\psi_{1,2}\right)=\frac{1}{2}\left|\nabla \psi_{1}\right|^{2}+\frac{1}{2}\left|\nabla \psi_{2}\right|^{2}+V\left(\psi_{1,2}\right),
$$




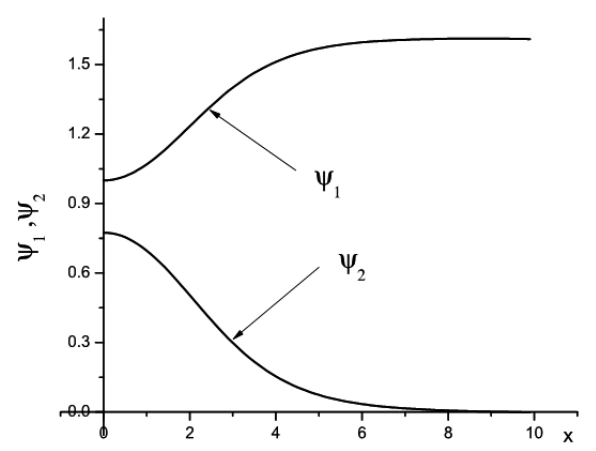

Fig. 1. The profiles of $\psi_{1,2}(x), \mu_{1}=1.61716, \mu_{2}=$ 1.49276 .

$$
\begin{aligned}
& V\left(\psi_{1,2}\right)=\frac{\lambda_{1}}{4}\left(\left|\psi_{1}\right|^{2}-\mu_{1}^{2}\right)^{2}-\frac{\lambda_{1}}{4} \mu_{1}^{4} \\
& +\frac{\lambda_{2}}{4}\left(\left|\psi_{2}\right|^{2}-\mu_{2}^{2}\right)^{2}-\frac{\lambda_{2}}{4} \mu_{2}^{4}+\frac{\lambda_{3}}{2}\left|\psi_{1}\right|^{2}\left|\psi_{2}\right|^{2} .
\end{aligned}
$$

Let us note that the energy functional is defined with accuracy of a constant. The constant is chosen as $-\frac{\lambda_{1}}{4} \mu_{1}^{4}-\frac{\lambda_{2}}{4} \mu_{2}^{4}$. The choice is made in such a way that to have only scattering terms without any other ones in Hamiltonian. In order to understand the physical sense of the obtained solution let us introduce the energy densities for both BEC's

$$
\begin{aligned}
& \varepsilon_{1}=\frac{1}{2}\left|\nabla \psi_{1}\right|^{2}+\frac{\lambda_{1}}{4}\left(\left|\psi_{1}\right|^{2}-\mu_{1}^{2}\right)^{2}-\frac{\lambda_{1}}{4} \mu_{1}^{4}, \\
& \varepsilon_{2}=\frac{1}{2}\left|\nabla \psi_{2}\right|^{2}+\frac{\lambda_{2}}{4}\left(\left|\psi_{2}\right|^{2}-\mu_{2}^{2}\right)^{2}-\frac{\lambda_{2}}{4} \mu_{2}^{4} .
\end{aligned}
$$

Taking into account the asymptotic behavior (10), (11) we see that the asymptotical behaviour of energy densities is

$$
\varepsilon\left(\psi_{1,2}\right) \rightarrow-\frac{\lambda_{1}}{4} \mu_{1}^{4}, \quad \varepsilon_{1} \rightarrow-\frac{\lambda_{1}}{4} \mu_{1}^{4}, \quad \varepsilon_{2} \rightarrow 0 .
$$

It means that we have the space filled with BEC described by $\psi_{1}$ and at the center there is a defect filled with BEC described by $\psi_{2}$. In Fig. 2 the profiles of the energy densities for both BEC's with $\psi_{1}$ and $\psi_{2}$ are presented.

We see that choosing the difference $E_{i}-v$ with some special values

$$
E_{i}-\frac{2 m_{i} V}{\hbar^{2} \psi^{3 / 2}(0)}=\lambda_{i} \mu_{i}^{2},
$$

we have the solution describing a defect filled with one kind of BEC on the background of the space filled with another kind of BEC. Equation (17) shows us that the regular solution presented in Figs. 1, 2 exist only for some special choice of $E_{1}$ and external trapping potential $V$ since $\mu_{i}$ are eigenfunctions.

From Fig. 2 we see that the BEC described by $\psi_{1}$ is cancelled from the space to a core. Formally the picture presented in Fig. 2 is similar to an Abrikosov vortex. The Abrikosov vortex is a vortex of supercurrent in a type-II superconductor. The supercurrent circulates around the normal (i.e. non-superconducting) core of the vortex. The circulating supercurrents induce magnetic fields

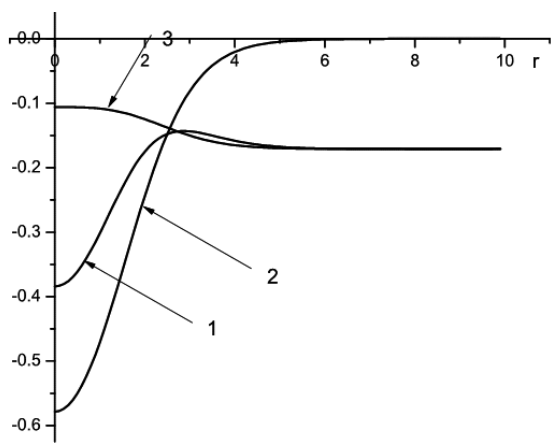

Fig. 2. The profiles of the dimensionless energy densities: curve 1 - the dimensionless energy density (12); curve 2 - the dimensionless energy density of the first BEC described by $\psi_{2},(14)$; curve 3 - the dimensionless energy density of the second BEC described by $\psi_{2},(15)$.

that are concentrated in a tube. Each vortex carries one thread of magnetic field with a single flux quantum $\Phi_{0}$. The similarity between the Abrokosov vortex and our solution for two BEC's is that the BEC described by $\psi_{1}$ is displaced from the space to a core since the energy density of this BEC tends to zero at the infinity but the energy density of both BEC's tends to non-zero value.

The profile for the dimensionless potential $\varepsilon\left(\psi_{1,2}\right)$ as the function of functions $\psi_{1,2}$ in Fig. 3 is presented. From this figure we see that it has two local and two global minima that gives rise to the regular solutions of Eqs. (5), (6).

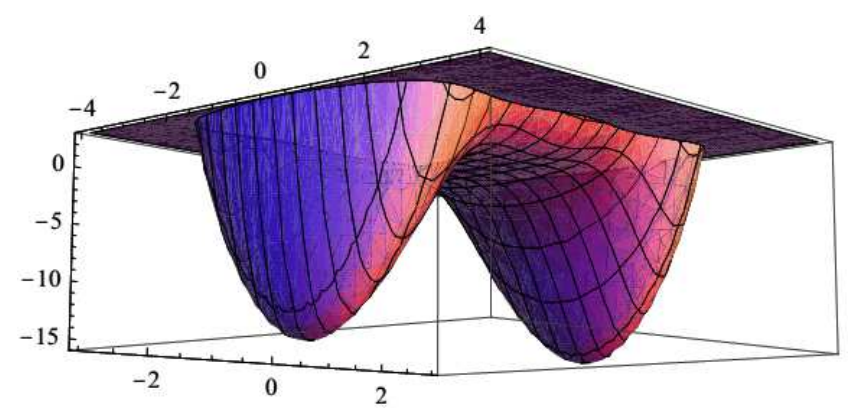

Fig. 3. The profile of dimensionless potential (12) as the function of $\psi_{1,2}$. One can see two local and two global minima.

The numerical investigations show that the solutions exist in a certain range of parameters $\lambda_{1,2}, \psi_{1}(0)$. Our investigation shows that the solution exist at least for $0.1<\lambda_{1}<0.5,1<\lambda_{2}<2,0.1<\psi_{2}(0)<2$.

\section{Droplet solution with three and two-body scattering terms and constant external trapping potential}

In this section we consider a droplet solution but BEC's have positive three-body $W_{11,22}\left|\psi_{1,2}\right|^{6}$ and negative two-body $-U_{11}\left|\tilde{\psi}_{1}\right|^{4}$ scattering terms on the right hand side (RHS) of Eqs. (18) and (19) [2]. 
In this case a two-component BEC can be described by the two-coupled equations (similar to GP) of the following form:

$$
\begin{aligned}
& \mathrm{i} \hbar \frac{\partial \tilde{\psi}_{1}}{\partial t}=\left(-\frac{\hbar^{2}}{2 m_{1}} \nabla^{2}+W_{11}\left|\tilde{\psi}_{1}\right|^{4}-U_{11}\left|\tilde{\psi}_{1}\right|^{2}\right. \\
& \left.\quad+U_{12}\left|\tilde{\psi}_{2}\right|^{2}+V\right) \tilde{\psi}_{1}, \\
& \mathrm{i} \hbar \frac{\partial \tilde{\psi}_{2}}{\partial t}=\left(-\frac{\hbar^{2}}{2 m_{2}} \nabla^{2}+W_{22}\left|\tilde{\psi}_{2}\right|^{4}-U_{22}\left|\tilde{\psi}_{2}\right|^{2}\right. \\
& \left.\quad+U_{21}\left|\tilde{\psi}_{1}\right|^{2}+V\right) \tilde{\psi}_{2},
\end{aligned}
$$

where the notations for $N_{i}$ are the same as in Sect. 2 . The resulting equations for the wave functions $\psi_{1,2}(\boldsymbol{r}, t)$ in dimensionless form can be written as

$$
\begin{aligned}
& \mathrm{i} \frac{\partial \psi_{1}}{\partial t}=\left(-\nabla^{2}+w_{11}\left|\psi_{1}\right|^{4}-u_{11}\left|\psi_{1}\right|^{2}\right. \\
& \left.\quad+u_{12}\left|\psi_{2}\right|^{2}+v\right) \psi_{1}, \\
& \mathrm{i} \frac{\partial \psi_{2}}{\partial t}=\left(-k \nabla^{2}+u_{22}\left|\psi_{2}\right|^{4}-u_{22}\left|\psi_{2}\right|^{2}\right. \\
& \left.\quad+u_{21}\left|\psi_{1}\right|^{2}+v\right) \psi_{2} .
\end{aligned}
$$

Here $k=m_{1} / m_{2}$ and we redefined $t / t_{0} \rightarrow t ; \boldsymbol{r} / l_{0} \rightarrow$ $\boldsymbol{r} ; \quad t_{0}=\frac{2 m_{1}}{\hbar \psi_{1}^{2 / 3}(0)} ; \quad l_{0}=\psi_{1}^{-1 / 3}(0) ; \quad \psi_{1,2}=\frac{\tilde{\psi}_{1,2}}{\psi_{1}(0)} ;$ $w_{i i}=\frac{2 m_{1} W_{i i} \psi_{1}^{10 / 3}(0)}{\hbar^{2}} ; \quad u_{i i}=\frac{2 m_{1} V_{i i} \psi_{1}^{4 / 3}(0)}{\hbar^{2}} ; u_{12,21}=$ $\frac{2 m_{1} U_{12,21} \psi_{1}^{4 / 3}(0)}{\hbar^{2}}$ and $v=\frac{2 m_{1} V}{\hbar^{2} \psi_{1}^{2 / 3}(0)}$. For the simplicity we will consider the case $k=1$. We are searching for a static spherical symmetric solution: $\psi_{1,2}(\boldsymbol{r}, t)=$ $\mathrm{e}^{-\mathrm{i} E_{1,2} t} \psi_{1,2}(r)$. In this case Eqs. (20), (21) are

$$
\begin{aligned}
& \psi_{1}^{\prime \prime}+\frac{2}{r} \psi_{1}^{\prime}=\psi_{1}\left[\lambda_{3} \psi_{2}^{2}+\lambda_{1}\left(\psi_{1}^{2}-\mu_{1}^{2}\right)\left(3 \psi_{1}^{2}-\mu_{1}^{2}\right)\right], \\
& \psi_{2}^{\prime \prime}+\frac{2}{r} \psi_{2}^{\prime}=\psi_{2}\left[\lambda_{3} \psi_{1}^{2}+3 \lambda_{2} \psi_{2}^{2}\left(\psi_{2}^{2}-\frac{2}{3} \mu_{2}^{2}\right)\right],
\end{aligned}
$$

where $3 \lambda_{1,2}=w_{11,22} ; \lambda_{3}=u_{12,21} ; u_{11}=\lambda_{1}\left(\mu_{1}^{2}+3\right)$; $\lambda_{1} \mu_{1}^{4}=v-E_{1} ; 2 \lambda_{2} \mu_{2}^{2}=u_{22}$ and $v-E_{2}=0$. Equations $(22),(23)$ are written in the form which is convenient for the numerical calculations. For the numerical calculations we choose the boundary conditions and parameters values as (7)-(9).

Again we search the solution as the nonlinear eigenvalue problem: $\mu_{1,2}$ are eigenvalues and corresponding functions $\psi_{1,2}$ are eigenfunctions. The profiles of functions $\psi_{1,2}$ in Fig. 4 are presented.

The asymptotic behavior of the functions $\psi_{1,2}$ for such two interacting BEC's is the following:

$$
\begin{aligned}
& \psi_{1} \approx \mu_{1}-\psi_{1, \infty} \frac{\mathrm{e}^{-r \sqrt{4 \lambda_{1} \mu_{1}^{4}}}}{r}, \\
& \psi_{2} \approx \psi_{2, \infty} \frac{\mathrm{e}^{-\mu_{1} r}}{r},
\end{aligned}
$$

where $\psi_{1,2, \infty}$ are constants.

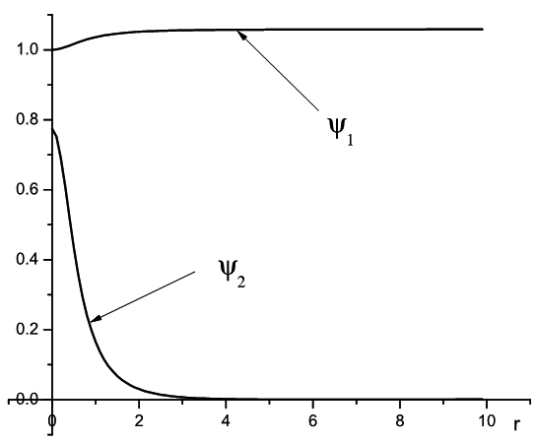

Fig. 4. The profiles of $\psi_{1,2}(x), \mu_{1}=1.0576294, \mu_{2}=$ 4.05682 .

One can consider Eqs. (22), (23) as the EulerLagrangian equations. Then the energy density has the form

$$
\begin{aligned}
& \varepsilon\left(\psi_{1,2}\right)=\frac{1}{2}\left|\nabla \psi_{1}\right|^{2}+\frac{1}{2}\left|\nabla \psi_{2}\right|^{2}+V\left(\psi_{1,2}\right), \\
& V\left(\psi_{1,2}\right)=\frac{\lambda_{1}}{2}\left|\psi_{1}\right|^{2}\left(\left|\psi_{1}\right|^{2}-m_{1}^{2}\right)^{2} \\
& \quad+\frac{\lambda_{2}}{2}\left|\psi_{2}\right|^{4}\left(\left|\psi_{2}\right|^{2}-m_{2}^{2}\right)+\frac{1}{2}\left|\psi_{1}\right|^{2}\left|\psi_{2}\right|^{2} .
\end{aligned}
$$

The energy densities for both BEC's are

$$
\begin{aligned}
& \varepsilon_{1}=\frac{1}{2}\left|\nabla \psi_{1}\right|^{2}+\frac{\lambda_{1}}{2}\left|\psi_{1}\right|^{2}\left(\left|\psi_{1}\right|^{2}-\mu_{1}^{2}\right)^{2}, \\
& \varepsilon_{2}=\frac{1}{2}\left|\nabla \psi_{2}\right|^{2}+\frac{\lambda_{2}}{2}\left|\psi_{2}\right|^{4}\left(\left|\psi_{2}\right|^{2}-\mu_{2}^{2}\right) .
\end{aligned}
$$

Taking into account the asymptotic behavior (24), (25) we see that at the infinity

$$
\varepsilon\left(\psi_{1,2}\right) \rightarrow 0 \text {. }
$$

It means that we have an energetic droplet filled with two interacting BEC's and trapped with the limitations

$$
u_{11}=\lambda_{1}\left(\mu_{1}^{2}+3\right), \quad v-E_{1}=\lambda_{1} \mu_{1}^{4}, \quad u_{22}=2 \lambda_{2} \mu_{2}^{2} .
$$

Equation (31) shows us that the regular solution presented in Figs. 1, 2 exists only for some special choice of $E_{1}$ and external trapping potential $V$ since $\mu_{i}$ are

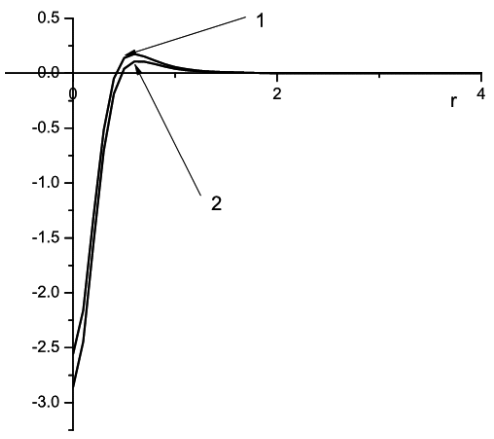

Fig. 5. The profiles of the dimensionless energy densities: the curve 1 - the dimensionless energy density (26) for both BEC's; the curve 2 - the dimensionless energy density (29) for the second BEC described by $\psi_{2}$. 


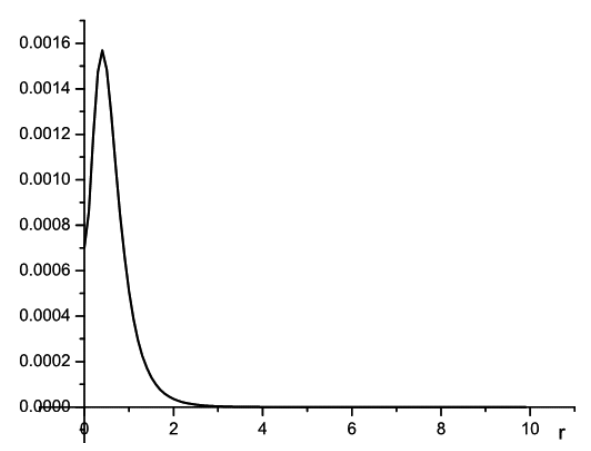

Fig. 6. The profile of the dimensionless energy density (28) for the first BEC described by $\psi_{1}$.

eigenfunctions. The energetic droplet means that although the number density $\left|\psi_{1}\right|^{1} \neq 0$ at the infinity the energy density $\varepsilon \rightarrow 0$.

In Figs. 5, 6 the profiles of the energy densities for both BEC's and for $\psi_{1}$ BEC and for $\psi_{2}$ BEC are presented.

The numerical investigations show that the solutions exist in a certain range of parameters $\lambda_{1,2}, \psi_{1}(0)$. Our investigation shows that the solution exists at least for $0.1<\lambda_{1}<0.2,1<\lambda_{2}<1.2,0.6<\psi_{2}(0)<0.8$.

\section{Energetic droplet solution with four and three-body scattering terms and without external trapping potential}

In this section we would like to consider the interaction between two BEC's where one BEC has a hypothesized positive strong four-body scattering term. The first BEC has four $P_{11}\left|\tilde{\psi}_{1}\right|^{8}$ and three-body $W_{11}\left|\tilde{\psi}_{1}\right|^{6}$ scattering terms but the second BEC has three and two-body scattering terms (analogously to Sect. 3). In order to obtain an energetic self-maintaining droplet without any external trapping potential we assume that there exists BEC with positive four-body scattering and negative three-body scattering.

In this case BEC's equations are

$$
\begin{aligned}
& \mathrm{i} \hbar \frac{\partial \tilde{\psi}_{1}}{\partial t}=\left(-\frac{\hbar^{2}}{2 m_{1}} \nabla^{2}+P_{11}\left|\tilde{\psi}_{1}\right|^{6}-W_{11}\left|\tilde{\psi}_{1}\right|^{4}\right. \\
& \left.\quad+U_{11}\left|\tilde{\psi}_{1}\right|^{2}+U_{12}\left|\tilde{\psi}_{2}\right|^{2}\right) \tilde{\psi}_{1}, \\
& \mathrm{i} \hbar \frac{\partial \tilde{\psi}_{2}}{\partial t}=\left(-\frac{\hbar^{2}}{2 m_{2}} \nabla^{2}+W_{22}\left|\tilde{\psi}_{2}\right|^{4}-U_{22}\left|\tilde{\psi}_{2}\right|^{2}\right. \\
& \left.\quad+U_{21}\left|\tilde{\psi}_{1}\right|^{2}\right) \tilde{\psi}_{2} .
\end{aligned}
$$

The resulting equations for the wave functions $\psi_{1,2}(\boldsymbol{r}, t)$ in dimensionless form can be written as

$$
\begin{aligned}
& \mathrm{i} \frac{\partial \psi_{1}}{\partial t}=\left(-\nabla^{2}+p_{11}\left|\psi_{1}\right|^{6}-w_{11}\left|\psi_{1}\right|^{4}+u_{11}\left|\psi_{1}\right|^{2}\right. \\
& \left.\quad+u_{12}\left|\psi_{2}\right|^{2}\right) \psi_{1}, \\
& \mathrm{i} \frac{\partial \psi_{2}}{\partial t}=\left(-k \nabla^{2}+w_{22}\left|\psi_{2}\right|^{4}-u_{22}\left|\psi_{2}\right|^{2}\right.
\end{aligned}
$$

$$
\left.+u_{21}\left|\psi_{1}\right|^{2}\right) \psi_{2} \text {. }
$$

Here $k=m_{1} / m_{2}$ and we redefined $t / t_{0} \rightarrow t ; \boldsymbol{r} / l_{0} \rightarrow \boldsymbol{r}$; $t_{0}=\frac{2 m_{1}}{\hbar \psi_{1}^{2 / 3}(0)} ; \quad l_{0}=\psi_{1}^{-1 / 3}(0) ; \quad \psi_{1,2}=\frac{\tilde{\psi}_{1,2}}{\psi_{1}(0)} ; p_{11}=$ $\frac{2 m_{1} \psi_{1}^{16 / 3}(0)}{\hbar^{2}} P_{11} ; w_{i i}=\frac{2 m_{1} \psi_{1}^{10 / 3}(0)}{\hbar^{2}} W_{i i} ; u_{i i}=\frac{2 m_{1} \psi_{1}^{4 / 3}(0)}{\hbar^{2}} U_{i i}$ and $u_{i j}=\frac{2 m_{1} \psi_{1}^{4 / 3}(0)}{\hbar^{2}} U_{i j}$. For the simplicity we will consider the case $k=1$. We are searching for a static spherical symmetric solution: $\psi_{1,2}(\boldsymbol{r}, t)=\psi_{1,2}(r)$. In this case Eqs. (34), (35) are

$$
\begin{aligned}
& \psi_{1}^{\prime \prime}+\frac{2}{r} \psi_{1}^{\prime}=\psi_{1}\left[\lambda_{3} \psi_{2}^{2}+\lambda_{1} \psi_{1}^{2}\left(\psi_{1}^{2}-\mu_{1}^{2}\right)\left(2 \psi_{1}^{2}-\mu_{1}^{2}\right)\right], \\
& \psi_{2}^{\prime \prime}+\frac{2}{r} \psi_{2}^{\prime}=\psi_{2}\left[\lambda_{3} \psi_{1}^{2}+3 \lambda_{2} \psi_{2}^{2}\left(\psi_{2}^{2}-\frac{2}{3} \mu_{2}^{2}\right)\right],
\end{aligned}
$$

where $2 \lambda_{1}=p_{11} ; 3 \lambda_{1} \mu_{i}^{2}=w_{11} ; \lambda_{1} \mu_{1}^{4}=u_{11} ; \lambda_{3}=u_{12}=$ $u_{21} ; 3 \lambda_{2}=w_{22}$; and $2 \lambda_{2} \mu_{1}^{2}=u_{22}$. Equations (36), (37) are written in the form which is convenient for the numerical calculations. For the numerical calculations we choose the boundary conditions and parameters values as in (7)-(9).

The solution is searched as the nonlinear eigenvalue problem: $\mu_{1,2}$ are eigenvalues and corresponding functions $\psi_{1,2}$ are eigenfunctions. We solve equations set (36), (37) numerically. The profiles of functions $\psi_{1,2}$ practically coincide with the profiles of the functions $\psi_{1,2}$ from Sect. 3 .

The asymptotic behavior of the functions $\psi_{1,2}$ is following:

$$
\begin{aligned}
& \psi_{1} \approx \mu_{1}-\psi_{1, \infty} \frac{\mathrm{e}^{-r \sqrt{2 \lambda_{1} \mu_{1}^{6}}}}{r}, \\
& \psi_{2} \approx \psi_{2, \infty} \frac{\mathrm{e}^{-\mu_{1} r}}{r},
\end{aligned}
$$

where $\psi_{1,2, \infty}$ are constants.

One can consider Eqs. (36), (37) as the EulerLagrangian equations. Then the dimensionless energy density has the form

$$
\begin{aligned}
& \varepsilon\left(\psi_{1,2}\right)=\frac{1}{2}\left|\nabla \psi_{1}\right|^{2}+\frac{1}{2}\left|\nabla \psi_{2}\right|^{2}+V\left(\psi_{1,2}\right), \\
& V\left(\psi_{1,2}\right)=\frac{\lambda_{1}}{4}\left|\psi_{1}\right|^{4}\left(\left|\psi_{1}\right|^{2}-\mu_{1}^{2}\right)^{2} \\
& \quad+\frac{\lambda_{2}}{2}\left|\psi_{2}\right|^{4}\left(\left|\psi_{2}\right|^{2}-\mu_{2}^{2}\right)+\frac{1}{2}\left|\psi_{1}\right|^{2}\left|\psi_{2}\right|^{2} .
\end{aligned}
$$

The energy densities for both BEC's are

$$
\begin{aligned}
& \varepsilon_{1}=\frac{1}{2}\left|\nabla \psi_{1}\right|^{2}+\frac{\lambda_{1}}{4}\left|\psi_{1}\right|^{4}\left(\left|\psi_{1}\right|^{2}-\mu_{1}^{2}\right)^{2}, \\
& \varepsilon_{2}=\frac{1}{2}\left|\nabla \psi_{2}\right|^{2}+\frac{\lambda_{2}}{2}\left|\psi_{2}\right|^{4}\left(\left|\psi_{2}\right|^{2}-\mu_{2}^{2}\right) .
\end{aligned}
$$

Taking into account the asymptotic behavior (38), (39) we see that at the infinity

$$
\varepsilon\left(\psi_{1,2}\right) \rightarrow 0, \quad \varepsilon\left(\psi_{1}\right) \rightarrow 0, \quad \varepsilon\left(\psi_{2}\right) \rightarrow 0 .
$$

It means that again we have an energetic droplet filled with two interacting BEC's and trapped with the limitations on parameters as follows: 
$w_{11}=3 \lambda_{1} \mu_{i}^{2}, \quad u_{11}=\lambda_{1} \mu_{1}^{4}, \quad u_{22}=2 \lambda_{2} \mu_{1}^{2}$.

It allows us to emphasize that the solutions obtained here exist for special kind of negative $-W_{11}\left|\psi_{1}\right|^{6}$ three-body, positive $U_{11}\left|\psi_{1}\right|^{4}$ two-body and negative $-U_{22}\left|\psi_{2}\right|^{4}$ two-body scattering terms.

The profiles of the dimensionless energy densities (40), (42), and (43) practically do not differ from the corresponding functions from Sect. 3 .

\section{Discussion and conclusions}

From the mathematical point of view regular solutions (having either a finite energy or at least finite values of the fields) for two interacting BEC's do exist only because the corresponding potential either (13) or (27) or (41) has local and global minima. If this potential has only a global minimum then following Derrick's theorem [13] such solution may exist in the dimension $1+1$ only $^{\dagger}$ and this solution is known as a kink ${ }^{\ddagger}$. It is interesting to compare the properties of presented here solution with the kink solution. The main difference is that the solution obtained here is topologically trivial whereas the kink is topological non-trivial solution. The topological non-triviality means that there exists an integer (a topological charge) that does not change by a small deviation of the solution. Usually the topological charge is characterized by the solution behavior at the infinity. Let us emphasize once again that the solutions obtained here are topologically trivial and they exist because the corresponding potential has both local and global minima only.

From the physical point of view the existence of presented solutions strongly depends on the existence of desired $n$-body interactions $(n=2,3,4)$ :

- The defect solution presented in Sect. 2 exists if the parameters $E_{1,2}$ and an external potential $V$ are linked with the relations (17).

- For the existence of the solution presented in Sect. 3 it is necessary to have a positive three-body and negative two-body scattering terms. In Ref. [2] arguments in favour of the existence of such interaction for one BEC are adduced. These arguments are based on results of Ref. [15] where the amplitude for the three-particle collisions is calculated. There it is shown that the contribution of this interaction to the energy density can dominate the contribution arising from the two-body collisions.

\footnotetext{
†Under the condition of absence of other fields: for instance, the t'Hooft-Polyakov monopole solution [14] is regular one and has only a global minimum but there exist additional fields — SU(2) Yang-Mills gauge fields.

¥It should be reminded that Derrick in his paper specifically states that his theorem is altered if there are multiple fields or higher derivatives involved.
}

Here we assume that such three-body interaction exist for two interacting BEC's, too. Additionally the parameters $E_{1,2}, U_{11,22}$ and the external potential should be linked with the relations (31).

- For the solution presented in Sect. 4 a hypothesized four-body interaction for the first BEC is assumed. Three-body interaction should be negative for the first BEC and positive one for the second BEC. Additionally the parameters $W_{11}, U_{11,22}$ should be tied with the expression (45).

- The droplet solutions from Sects. 3 and 4 are an equilibrium state between attractive and repulsive interaction terms and have zero energy density at the infinity.

Now we would like to discuss the physical interpretation of the obtained solutions. The interpretation strongly depends on the asymptotic value of the energy densities. In the case of non-zero value we have the space filled with one Bose-Einstein condensate $\psi_{1}$ and on the background of this condensate there is a spherically symmetric defect (cavity) filled with another Bose-Einstein condensate $\psi_{2}$. The asymptotic behavior of both condensates is different: for $\psi_{1}$ condensate the asymptotic value is non-zero but for $\psi_{2}$ condensate the asymptotic value is zero. The same is valid for both energy densities. If the asymptotic value is zero we have energetic droplet solution: there exists a ball filled with two BEC's. There are two possibilities: either the energetic droplet is trapped with a constant external potential (Sect. 3) or not trapped without any external potential (Sect. 4). Let us note that for the solution presented in Sect. $4\left|\psi_{1}\right|^{2} \neq 0$ at the infinity but the energy density tends to zero. Such situation can be called as energetic droplet.

Numerical calculations show that most likely the existence of a regular solution depends on the potential form only: whether has it or not local and global minima simultaneously. In this connection one can mention that similar solutions have been found for a scalar model of a glueball [16]. In this model the Lagrangian from SU(3) gauge theory by some manner can be approximated by a Lagrangian with two scalar fields. These two scalar fields approximately describe 2th and 4th Green functions (i.e. the correlation between fields in two or four points in the spacetime) of $\mathrm{SU}(3)$ gauge fields. The first scalar field describes a gauge field belonging to subgroup $\mathrm{SU}(2) \subset \mathrm{SU}(3)$ and the second scalar field describes fields belonging to a coset $\mathrm{SU}(3) / \mathrm{SU}(2)$. The kinetic terms from the initial Lagrangian gives rise to kinetic terms for both scalar fields. The terms like $A_{\mu}^{B} A_{\nu}^{C} A_{\rho}^{D} A_{\sigma}^{E}$ give rise to terms like $\left(\psi_{i}^{2}-\psi_{i ; \infty}^{2}\right)^{2}$ in corresponding potentials for the scalar fields $\psi_{i}(i=1,2)$. Here $A_{\mu}^{B}$ is the $\mathrm{SU}(3)$ gauge potential. The difference between the potential (26) and the potential obtained in Ref. [16] is that the potential (26) is the polynomial of 6 th order whereas the potential from [16] is polynomial of 4 th order. But in both cases corresponding potentials have local and global 
minima that leads to the existence of a regular solution. In Ref. [2] a similar construction is considered and following conclusion is made: ". . a dilute Fermi-Dirac droplet will behave very much like a nuclear system".

Finishing the comparison of obtained here solution with the solution obtained for the scalar model of a glueball we see that there is clear analogy between both solutions. It allows us to draw interesting and useful analogy between physical objects from high energy physics and BEC's physics: (a) the defect solution is similar to a cavity on the background of the space filled with a nonzero gluon condensate; (b) the droplet solution is similar to a glueball.

Another important feature of the solution obtained here is following. In Ref. [17] it was shown that under certain conditions, the system of two BEC's has an almost degenerate ground state which is separated from the excited levels by an energy gap. The atomic ensemble then behaves like a two-level system that could be used to encode a qubit. The authors have offered the idea of using these two many-body states to encode a qubit and use it for quantum computation. One can use the defect/droplet obtained here as an elementary element (quantum gate) for creating the qubit device offered in Ref. [17].

Finally we would like to list the main features of the solutions obtained here:

- The presented defect/droplet solutions exist for two interacting BEC's only.

- The solutions exist for special choice of the BEC's potential: it should have local and global minimum only.

- The solutions are eigenfunctions for nonlinear differential equations. Consequently some physical parameters must have well defined values (external potential, coefficients in front of $n$-body scattering terms and so on).

- The energetic droplet solution without any external trapping potential exists in the case of very strong interaction between atoms of BEC only. The energetic droplet solution presented here does exist if there is a positive four-body scattering term of atoms for one BEC.

- The defect solution exists for two interacting BEC's but with some special choice of parameters $E_{i}$ and external trapping potential.

\section{Acknowledgments}

I am grateful to the Research Group Linkage Programme of the Alexander von Humboldt Foundation for the support of this research and the grants No. 378 in fundamental research in natural sciences by the Ministry of Education and Science of Kazakhstan. Special thanks for A. Avdeenkov for the fruitful discussion.

\section{References}

[1] T.L. Ho, V.B. Shenoy, Phys. Rev. Lett. 77, 3276 (1996); B.D. Esry, C.H. Greene, J.P. Burke, J.L. Bohn, Phys. Rev. Lett. 78, 3594 (1997); H. Pu, N.P. Bigelow, Phys. Rev. Lett. 80, 1130 (1998).

[2] A. Bulgac, Phys. Rev. Lett. 89, 050402 (2002).

[3] C.-C. Chien, F. Cooper, Phys. Rev. A 87, 045602 (2013).

[4] P.F. Bedaque, E. Berkowitz, S. Sen, J. Phys. B, At. Mol. Opt. Phys. 45, 225301 (2012).

[5] R. Wei, E. Mueller, Phys. Rev. A 84, 063611 (2011).

[6] V. Ramesh Kumar, R. Radha, M. Wadati, Phys. Lett. A 374, 3685 (2010).

[7] L.Q. Yan, L.Z. Dong, Y.S. Fang, L. Lu, F.G. Sheng, Chin. Phys. B 19, 080501 (2010).

[8] Deng-Shan Wang, Xing-Hua Hu, W.M. Liu, Phys. Rev. A 82, 023612 (2010).

[9] B.B. Baizakov, A.M. Kamchatnov, M. Salerno, J. Phys. B, At. Mol. Opt. Phys. 41, 215302 (2008).

[10] U. Al Kawaja, H. Stoof, Nature 411, 918 (2001); J. Ruostekoski, J.R. Anglin, Phys. Rev. Lett. 86, 3934 (2001); C.M. Savage, J. Ruostekoski, Phys. Rev. Lett. 91, 010403 (2003); J. Ruostekoski, Phys. Rev. A 70, 041601 (2004).

[11] K.J.H. Law, P.G. Kevrekidis, L.S. Tuckerman, Stable Vortex-Bright Soliton Structures in Two-Component Bose Einstein Condensates, arXiv:1001.4835.

[12] Xiao-Fei Zhang, Xing-Hua Hu, Xun-Xu Liu, W.M. Liu, Phys. Rev. A 79, 033630 (2009).

[13] G.H. Derrick, J. Math Phys. 5, 1252 (1964).

[14] G. 't Hooft, Nucl. Phys. B 79, 276 (1974); A.M. Polyakov, J. Exp. Theor. Phys. 41, 988 (1975).

[15] V. Efimov, Sov. J. Nucl. Phys. 29, 546 (1979).

[16] V. Dzhunushaliev, SU(3) Glueball Gluon Condensate, arXiv:1110.1427 [hep-ph].

[17] J.I. Cirac, M. Lewenstein, K. Molmer, P. Zoller, Phys. Rev. A 57, 1208 (1998). 